\title{
Estudio comparativo de la síntesis de nanopartículas de magnetita monodispersas
}

\author{
J.J. Atoche Medrano, J. A. Huamani Coaquira \\ Universidad de Brasília, Campus Universitário Darcy Ribeiro, Brasília - CEP 70910-900
}

DOI: https://doi.org/10.33017/RevECIPeru2015.0001/

\section{Resumen}

Actualmente el tipo de nanopartículas magnéticas más estudiados son los de estructura cúbica, espinela inversa, porque estos materiales presentan características de gran interés y sus posibles aplicaciones ya que facilitan la construcción de sistemas más complejos. Debemos considerar que debido a la presencia de metales de transición en la superficie de las nanopartículas es que se dan las condiciones para poder funcionalizarlas con otras moléculas a través de grupos funcionales complejos obteniendo materiales con características polares o apolares, dependiendo del tipo de aplicación que se desee utilizar. Teniendo en consideración que cuando se trabaja con sistemas biológicos las nanopartículas son detectadas por el sistema retículo endotelial (SRE), que a través de los macrófagos son los encargados de eliminar algún cuerpo extraño inerte que pudiera estar en el organismo. De esta manera, existe una necesidad de funcionalizar las nanopartículas obtenidas antes de ser administradas en el organismo para evitar ser reconocidas por el SRE. Esta funcionalización es responsable por evitar la aglomeración de las mismas permitiendo que ellas permanezcan en suspensión estable (coloides magnéticos) que pueden ser conducidos a través de campos magnéticos externos. En este trabajo, nosotros mostramos detalladamente los resultados obtenido en la mejora de la ruta de síntesis de un sistema de nano partículas en forma de ferrofluido de magnetita $\left(\mathrm{Fe}_{3} \mathrm{O}_{4}\right)$ utilizando el método de descomposición térmica y comparamos nuestros resultados respecto a otra ruta de síntesis para sistema nano particulados llamado método de co-precipitación química. Para poder medir el tamaño, así como conocer las propiedades morfológicas y estructurales de las nanopartículas se procedió a la caracterización de nanopartículas obtenidas por los métodos de descomposición térmica y co-precipitación química a través de microscopia electrónica de transmisión (MET). Se encontró una distribución de tamaños con un promedio de $8 \mathrm{~nm}$ y polidispersión de 0.14 . Estos resultados fueron corroborados por los resultados obtenidos mediante análisis de patrones de difracción de rayos X. La estabilidad del ferrofluido obtenido fue medida usando la técnica conocida como DLS (Dynamic Light Scattering), donde fue encontrado un valor de $42.8 \mathrm{mV}$, que está dentro del valor esperado para un sistema estable, considerando que para un sistema nanopartículado el valor de Zetasiser arriba de $30 \mathrm{mV}$ representa una estabilidad de la suspensión acuosa. Al final de las medidas de caracterización se realizó la medida del valor del potencial de hidrógeno $(\mathrm{pH})$ mediante un $\mathrm{pH}$-metro, para estudiar la biocompatibilidad que presenta nuestra muestra de ferrofluido ya que nuestro interés es que este ferrofluido pueda ser usado como vehículo para direccionar principios activos o fármacos sobre una región específica en el organismo. Así después de realizada la medición fue encontrado un valor del $\mathrm{pH}$ de 7.23 lo que evidencia un sistema biocompatible para posibles aplicaciones biológicas.

Descriptores: Descomposición térmica, co-precipitación, ferrofluido, nanopartículas, microscopia electrónica de transmisión.

\section{Abstract}


Currently the most studied type of magnetic nanoparticles are of cubic structure, inverse spinel, because these materials have very interesting features and possible applications since they facilitate building more complex systems. We must consider that due to the presence of transition metal on the surface of nanoparticles is that the conditions for funcionalizarlas with other molecules through complex functional groups obtaining materials with polar or apolar characteristics, depending on the type of implementation that want to use. Considering that when working with biological systems nanoparticles are detected by the reticuloendothelial system (RES). This functionalization is responsible for preventing agglomeration there of allowing them to remain in stable suspension (magnetic colloids) that can be driven by external magnetic fields. In this work, we show in detail the results obtained in improving the synthesis route system in the form of nanoparticles ferrofluid magnetite (Fe3O4) using the method of thermal decomposition and compare our results with respect to another synthesis route to nano particulate system method called chemical coprecipitation. To measure the size as well as knowing the morphological and structural properties of nanoparticles proceeded to the characterization of nanoparticles obtained by the methods of thermal decomposition and chemical co-precipitation through transmission electron microscopy (TEM). Size distribution averaging $8 \mathrm{~nm}$ and polydispersity of 0.14 was found. These results were corroborated by the results obtained by analyzing patterns of X-ray diffraction. The stability of the ferrofluid obtained was measured using the technique known as DLS (Dynamic Light Scattering), where it was found a value of $42.8 \mathrm{mV}$, which is within the expected value for a stable system, whereas for a nanoparticle system Zetasiser value above $30 \mathrm{mV}$ represents a stability of the aqueous suspensión. At the end of characterization measures the extent of the value of the potential of hydrogen $(\mathrm{pH})$ was performed using a $\mathrm{pH}$ meter, to study our sample having biocompatibility ferrofluid as our interest is that the ferrofluid could be used as vehicle for active ingredients or drugs addressing to a specific region in the body. And after completion of the measurement it was found a $\mathrm{pH}$ of 7.23 which shows a biocompatible system to possible biological applications.

Keywords: Thermal decomposition, co-precipitation, ferrofluid, nanoparticles, transmission electron microscopy.

\section{Introducción}

Dentro del conjunto de las nanopartículas que se han venido estudiando en los últimos años, las nanopartículas magnéticas son una de las que despiertas mayor interés [1]. Más un aspecto interesante es que ellas han sido estudiadas extensamente a partir de los años 40, pero curiosamente fue recién en los años 50 , cuando a partir de los estudios realizados se observó que estas tenían elevada resistividad eléctrica [2], lo que despertó un interés pues a partir de esta propiedad se podrían evitar el efecto de las corrientes parásitas (Foucault). En ese sentido, estas nanopartículas resultan ser de interés por ejemplo en dispositivos que trabajan con altas frecuencias (por ejemplos radares o transformadores que poseen núcleos de ferrita). Pudiendo ser empleados también en dispositivos de almacenamiento de dispositivos de información [3], así como pigmentos de elevada estabilidad térmica y química [4]. Todos los aspectos que estamos mencionando están envueltos con lo que se conoce como nanociencia, cuyos principios están marcados por diversos hallazgos relacionados con las diferentes características físicas y químicas que presentan los materiales con escala nanométrica respecto a los materiales bulk de igual composición [5]. Estas nuevas propiedades que adquieren se deben básicamente a dos aspectos: El incremento en la relación superficie/volumen debido a la elevada área superficial en este rango de tamaños y la modificación de los estados electrónicos del material debido al confinamiento cuántico debido al tamaño reducido de las partículas [6]. A partir de lo expuesto anteriormente encontramos que las ferritas de composición $\mathrm{MFe}_{2} \mathrm{O}_{4}$ (donde $\mathrm{M}$ representa un metal de transición divalente como por ejemplo $\mathrm{Co}, \mathrm{Mn}, \mathrm{Ni}, \mathrm{Zn}$ ), muestran un vasto estudio relacionado a sus interesantes propiedades magnéticas cuando son obtenidas a escala nanométrica $[7,8]$.

Cuando consideramos nanopartículas debido su reducido tamaño, estas muestran una energía asociada que hace que determinadas distribuciones catiónicas desordenadas correspondientes a una estructura espinela mixta (directa e inversa), sean estables [9]. Considerando que estás partículas monodominio (partículas que debido a su reducido tamaño no es energéticamente favorable la formación de paredes de Bloch), mostrando un comportamiento superparamagnetico arriba de la temperatura de bloqueo (TB) [10].

Así, de esta manera existe un creciente interés en obtener diversos tipos de nanopartículas con propiedades adecuadas para cada fin. Actualmente se están desarrollando diversas técnicas para la 
obtención de estos productos, considerando la homogeneidad, tamaño, forma, estabilidad, aglomeración, etc. El objetivo de este trabajo es determinar el efecto sobre las propiedades de nanoparticulas de magnetita preparadas por los dos métodos de síntesis ampliamente usados (coprecipitación química y descomposición térmica).

\section{Material y métodos}

Todos los reactivos químicos utilizados durante el transcurso del trabajo fueron de grado puro para análisis, y se usaron sin purificaciones adicionales. Todos los reactivos usados fueron adquiridos por la marca Sigma-Aldrich. Los reactivos empleados para el proceso de síntesis por el método de coprecipitación química fueron: $\mathrm{NaOH}(0.32 \mathrm{~mol})$; cloruro férrico hexahidratado $\mathrm{Fe} 2 \mathrm{Cl} 3.6 \mathrm{H} 2 \mathrm{O}$ (0.01 $\mathrm{mol})$; cloruro de manganeso $\mathrm{MnCl} 2(0.013 \mathrm{~mol})$. Se usó agua destilada a lo largo de todo el proceso de síntesis.

Para el método de descomposición térmica los reactivos usados fueron: $\mathrm{Fe}(\mathrm{acac}) 3$ (2 mmol), 1,2hexadecanodiol $(10 \mathrm{mmol})$, benzyl eter $(20 \mathrm{ml})$, ácido oleico $(6 \mathrm{mmol})$, oleylamina $(6 \mathrm{mmol})$, gas nitrógeno para controlar la atmósfera. Fue utilizado un balón de vidrio pirex de $100 \mathrm{ml}$ con 3 bocas. Un agitador magnético con manta térmica para controlar la temperatura.

Para caracterización usando micrografía fue usado un microscopio electrónico de transmisión de la Universidad de Brasília, Instituto de Ciencias Biológicas. Se usó disolución de las muestras de $1: 1000$ y $1: 500$, las cuales fueran depositadas sobre las grade de cobre (200 mesh) recubiertas con formvar.

Las muestras fueron caracterizadas por DRX para determinar su composición de fases y estimar el tamaño medio de los cristalitos. Los patrones de DRX se registraron con un equipo D8 Advance (Bruker) usando geometría Bragg-Brentano; radiación $\mathrm{KaCu}(\lambda=1,54183 \AA)$, monocromador secundario de grafito; rango de barrido entre $20^{\circ}$ y $80^{\circ}$, y paso $0,05^{\circ}$.

La estabilidad del ferrofluido fue medido usando un equipo Zetasizer Malvern en el Instituto de Ciencias Biológicas de la Universidad de Brasília. Todas las medidas fueron realizadas por triplicado y a temperatura ambiente. El valor del potencial Hidrógeno fue medido utilizando un $\mathrm{pH}$ metro en el Instituto de Ciencias Biológicas de la Universidad de Brasília, donde previamente el instrumento fue calibrado con solución tampón y seguidamente se realizó las medidas a temperatura ambiente. Todas las medidas fueron realizadas por triplicado.

\section{Síntesis de nanopartículas por co- precipitación química}

Inicialmente se mesclan el cloruro férrico y el cloruro de manganeso en un Becker de $250 \mathrm{ml}$ adicionando $50 \mathrm{ml}$ de agua destilada e agitación magnética vigorosa para completar la disolución de los componentes, seguidamente el sistema es calentado hasta conseguir una temperatura de $70{ }^{\circ} \mathrm{C}$, en ese momento es adicionado la base de hidróxido de sodio sobre nuestra solución, observándose al momento el cambio de color para un color oscuro, característico de la formación de ferrita, el sistema fue dejado en agitación con temperatura controlado por 15 minutos y después fue dejado enfriar en presencia de un campo magnético externo para conseguir un precipitado hasta llegar a la temperatura ambiente, para luego realizar el lavado (3 veces) y retirar el sobrenadante. Finalmente el precipitado es llevado a la estufa y dejado secar a $70^{\circ} \mathrm{C}$ durante 24 horas.

\subsection{Síntesis de nanopartículas por descomposición térmica}

Se colocaron todos los reactantes dentro de un balón de vidrio de 3 bocas, se colocó el flujo de nitrógeno, el tubo para resfriar el sistema y la salida de vapor. Seguidamente, se procedió a elevar la temperatura hasta $220{ }^{\circ} \mathrm{C}$, siempre manteniendo una agitación constante y se dejó por 30 minutos para, después, nuevamente elevar la temperatura hasta unos $260{ }^{\circ} \mathrm{C}$, dejándose nuevamente por 30 minutos. Después de esto, se dejó enfriar el sistema hasta llegar a la temperatura ambiente. Finalmente, el producto fue lavado con etanol (3 veces) y centrifugado para luego ser dispersado en hexano.

\section{Resultados y discusión}

La síntesis de co-precipitación química para la obtención de nanopartículas magnéticas es un método más sencillo y de bajo costo que permite obtener un rendimiento considerable. En el presente trabajo fueron realizados análisis de difracción de rayos x para las dos rutas de síntesis seguidas.

En la Figura 1 se muestra el difractograma de rayos $X$ de nanopartículas de magnetita sintetizadas por co-precipitación química. Fue determinada la presencia de una sola fase cristalina del tipo espinelio. El difractograma mostrado es similar al reportado en la Ref. 11 para nanopartículas de 
ferrita, las cuales fueron preparadas por el método de co-precipitación. En el trabajo reportado en la Ref.12 se muestra un difractograma de rayos $X$ con picos que presentan una buena similaridad al obtenido en nuestro trabajo, evidenciando de esta manera que nuestra muestra presenta una estructura cristalina propia de magnetita.

La determinación de la distribución de tamaño físico de las nanoparticulas fue realizada mediante microscopia electrónica de transmisión con un aumento de 50 mil veces su tamaño. En la Figura 2 puede observarse que estas nanopartículas presentan una polidispersión acentuada, además que la forma que presentan las partículas no es uniforme. Según el trabajo reportado en la Ref. 13 se obtuvieron resultados similares, donde las nanopartículas de magnetita poseen un tamaño arriba de los $50 \mathrm{~nm}$ y bastante polidisperso. El tamaño grande e la grande polidispersión podría ser consecuencia de la velocidad de agitación, que en este caso fue de 800 revoluciones por minuto, y también de la temperatura en la cual es realizada la síntesis como sugiere el trabajo reportado en la Ref. 14.

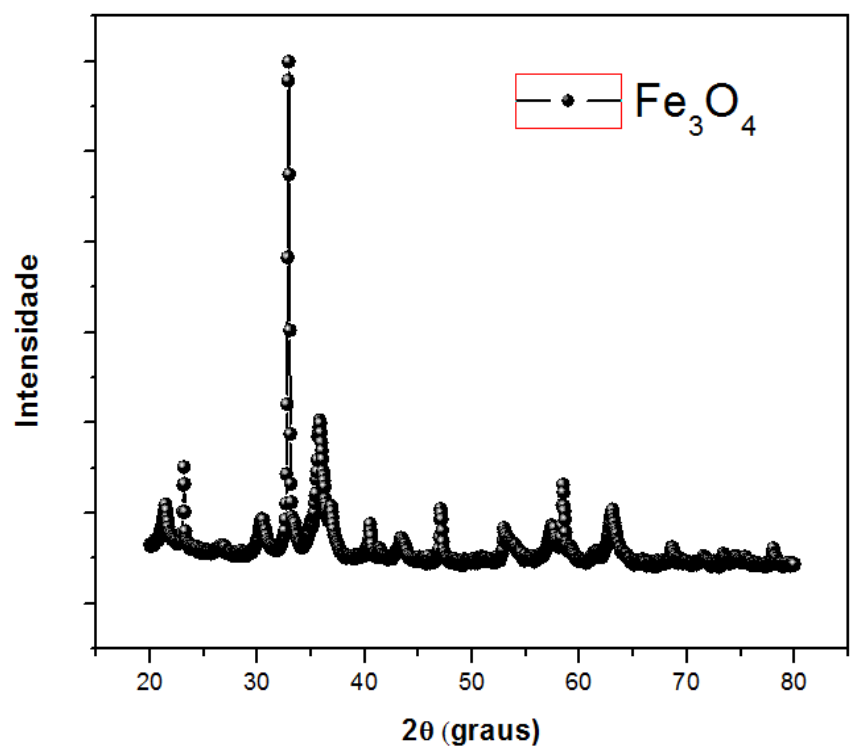

Figura 1. Patrón de difracción de rayos $X$ de magnetita obtenida por co-precipitación química.

Ya en el trabajo reportado en la Ref.15, los autores mencionan que el tamaño de las nanopartículas puede verse afectado por la velocidad con la cual es adicionada la base.

En nuestro caso, como fue mencionado en la metodología, la base fue adicionada rápidamente sobre la solución acuosa, este factor es posible que sea el responsable por el tamaño de las nanopartículas obtenidas.
En la Figura 3, se muestra el patrón de difracción de las nanopartículas obtenidas por descomposición térmica, en este caso observamos que el difractograma al ser analizado vía refinamiento de Rietveld usando el programa GSAS coincide con el padrón esperado para la magnetita.

Según el trabajo reportado en la Ref. 16, los resultados muestran concordancia con el espectro de difracción de rayos $X$ que ellos obtuvieron a partir de la síntesis por descomposición térmica usando ácido oleico como surfactante.

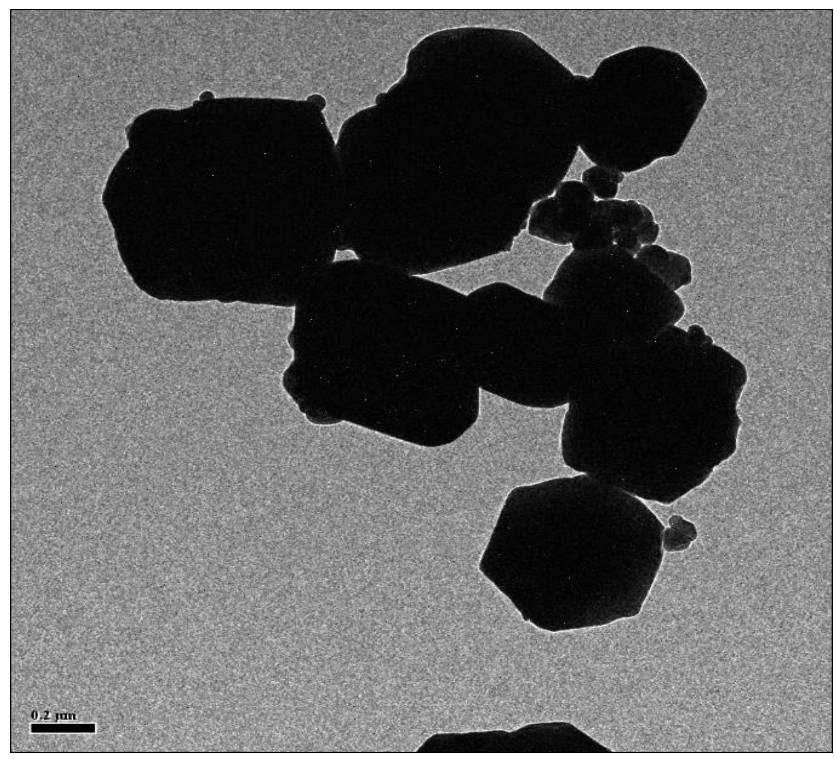

Figura 2. Micrografía electrónica de transmisión de nanopartículas de magnetita obtenidas por coprecipitación química.

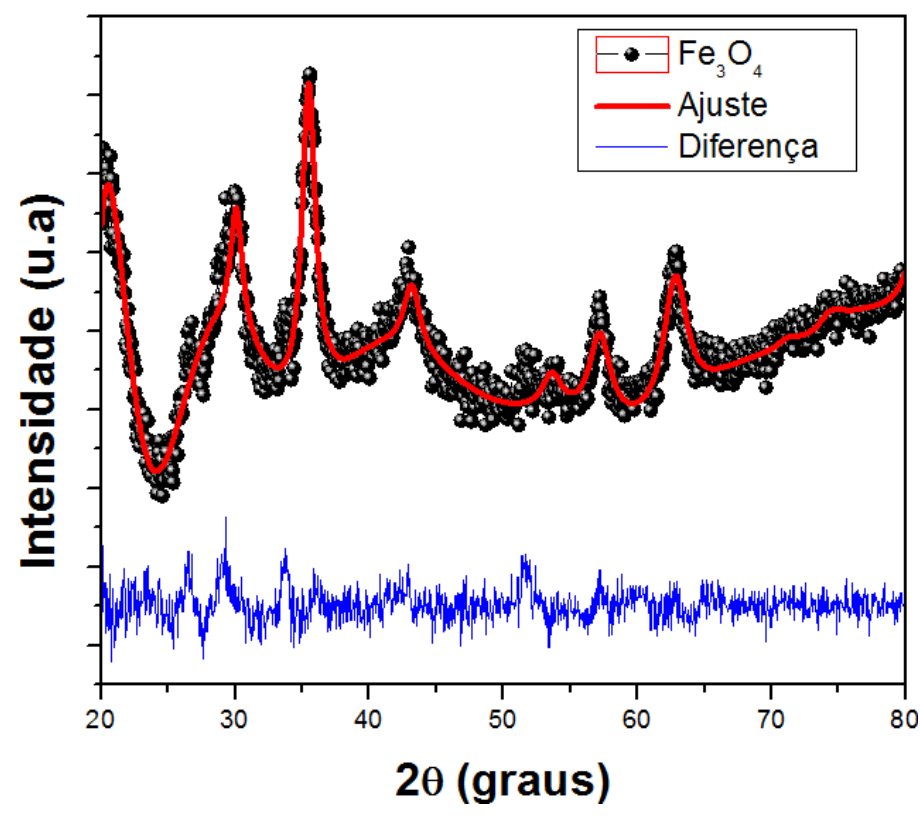

Figura 3. Patrón de difracción de rayos $X$ de nanopartículas de magnetita obtenidas por 
decomposición térmica refinado por el método de Rietveld usando programa GSAS.

El patrón de nuestro difractograma también muestra una consistencia respecto a la estructura espinela inversa reportada en la Ref. 17, considerando que también fue usado en la síntesis surfactante oleylamina y ácido oleico, además de usar el agente reductor 1,2 hexadodecanodiol para obtener nanopartículas con las mismas características que fueron usadas en nuestro trabajo.

La imagen por microscopía electrónica de transmisión presentada en la Figura 4, muestra la morfología de las nanopartículas. Como puede observarse, las nanoparticulas muestran una tendencia de forma esférica las cuales están separadas unas de las otras por la presencia del surfactante lo que evita la aglomeración de las nanopartículas.

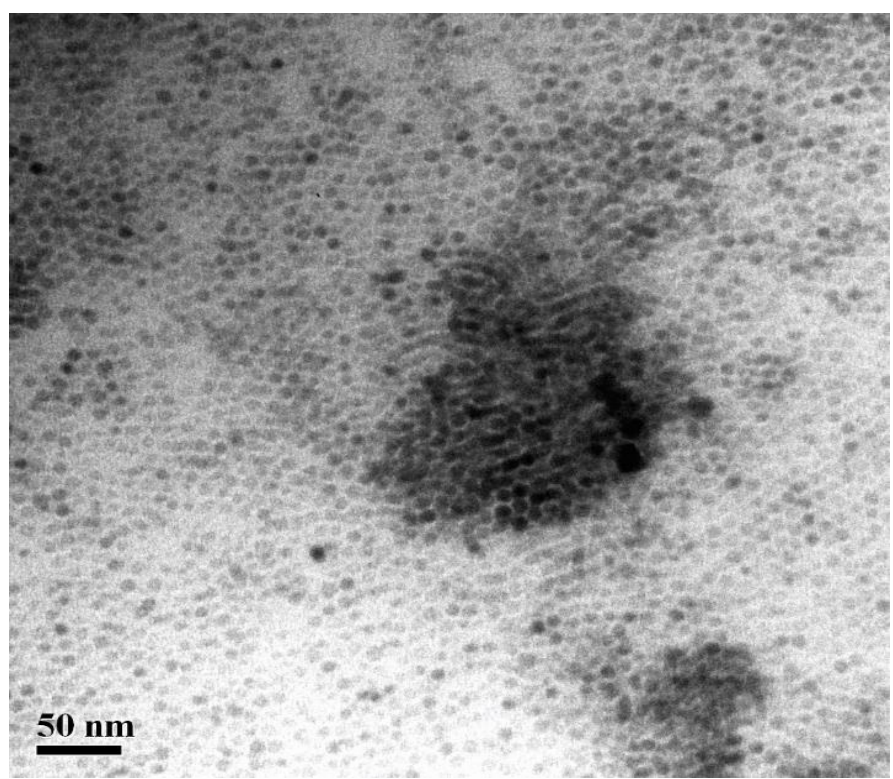

Figura 4. Micrografía electrónica de transmisión de nanopartículas de magnetita sintetizadas por descomposición térmica.

El trabajo reportado en la Ref. 18 muestra imágenes de nanopartículas muy semejantes a las nuestras con ligeras diferencias. Estas diferencias radicarían en la ruta de síntesis que ellos usaron. Los autores utilizaron el disolvente fenileter, el cual es muy importante para obtener tamaño pequeño y controlar la morfología de las nanopartículas.

Finalmente el resultado para nuestro sistema de ferrofluido mostro concordancia con el valor del $\mathrm{pH}$ medido ( $\mathrm{pH}=7.23$ ), como fue mostrado en los resultados del trabajo desarrollado por los autores en la Ref. 20, los cuales utilizaron ácido oleico como surfactante durante la síntesis de las nanopartículas.

En la Figura 5 mostramos los resultados del conteo de los tamaños de las nanopartículas usando el programa Image Pro Plus, mediante un histograma. Se contaron $\mathrm{N}=800$ nanopartículas para obtener el tamaño y el histograma fue montado usando el método de Sturges.

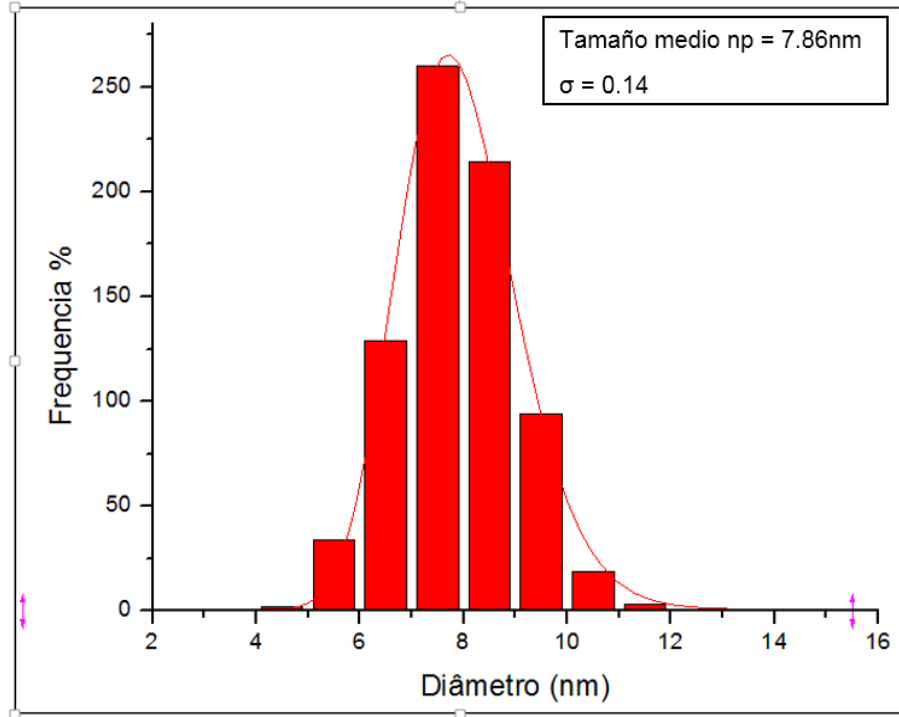

Figura 5. Distribución de tamaño de nanopartículas de magnetita sintetizadas por descomposición térmica. El histograma fue montado usando el método de Sturges. La línea continua representa el ajuste con función Lognormal.

El análisis del histograma usando una distribución Lognormal muestra una estrecha distribución de tamaños (polidispersión, $\sigma=0.14$ ). Podemos apreciar que en este caso el resultado indica que las nanopartículas de magnetita obtenidas tiene una distribución uniforme de tamaño es decir es un sistema monodisperso.

Nuestros resultados obtenidos por microscopia electrónica de transmisión también están acorde con los resultados reportados en la Ref. 19, donde se muestra un tamaño de nanopartículas próximas a 8 $\mathrm{nm}$, donde al igual que nuestros resultados se puede apreciar el patrón de ordenamiento en la distribución de las nanopartículas, aduciendo una formación cristalina característica de estructuras espinelas.

Estos resultados son bastante interesantes en materiales nanoestructurados porque permite aplicaciones en sistemas biológicos, ya que al ser administrado en el organismo debido a la forma esférica que las partículas presentan, el desplazamiento es más uniforme, y por su tamaño 
reducido las mismas presentan propiedades que las tornan más reactivas debido al alto valor de área superficial.

\section{Conclusiones}

A partir de los resultados obtenidos podemos concluir: Cada una de las rutas seguidas presenta ventajas y limitaciones respecto una de otra. Por ejemplo, costos de materiales, tiempo, preparación de las muestras para su posterior análisis y caracterización respectiva. Tamaños pequeños $(8 \mathrm{~nm})$ son obtenidos al usarse el método de descomposición térmica y tamaños de $\sim 50 \mathrm{~nm}$ son obtenidos al usarse el método de co-precipitación. Es importante tener en consideración cuales son las posibles aplicaciones que se están buscando del producto final para una correcta decisión a la hora de sintetizar las nanopartículas de tamaño e morfología específicas. La ocurrencia de interacciones partícula-partícula puede ser controlada variando el método de síntesis y los reactivos usados. El sistema obtenido a través del método de descomposición térmica, permite aplicaciones en el área de Nanobiotecnologia debido a las propiedades que presenta (monodispersión, estabilidad, biocompatibilidad).

\section{Agradecimientos}

Agradecemos el apoyo brindado a la Coordinación de perfeccionamiento de personal de nivel superior CAPES, por el apoyo económico para el desarrollo de la investigación. Al personal técnico de los diferentes laboratorios de la Universidad de Brasília por la disponibilidad para el uso de equipos. Al grupo de investigación de Nanociencia y Nanobiotecnologia así como al Núcleo de Física Aplicada de la Universidad de Brasília por todo el apoyo brindado durante el desarrollo de este trabajo.

\section{Referencias}

[1] A. Figuerola; R. Di Corato; L. Manna; T. Pellegrino. From iron oxide nanoparticles towards advanced iron-based inorganic materials designed for biomedical applications. Pharmacological Research. 62 (2010) 126-143.

[2] I. H. Gul; A. Z. Abbasi; F. Amin; M. Anis-Urrehman; A. Maqsood. Structural, magnetic and electrical properties of $\mathrm{Co} 1-\mathrm{x} \mathrm{ZnxFe}_{2} \mathrm{O}_{4}$ synthesized by co-precipitation method. Journal of Magnetism and Magnetic Materials. 311 (2007) 494-499.

[3] G. Reiss; A. Hutten. Magnetic nanoparticles:
Applications beyond data storage. Nature Material. 4 (2005) 725-726.

[4] A. S. Teja; P.Y. Koh. Synthesis, properties, and applications of magnetic iron oxide nanoparticles. Progress in Crystal Growth and Characterization of Materials. 55 (2009) 22-45.

[5] C. N. R. Rao; G. U. Kulkarni; P. J. Thomas; P. P Edwards. Size-Dependent Chemistry: Properties of Nanocrystals. Chemistry - A European Journal. 8 (2002) 28-35.

[6] N. Lee; T. Hyeon. Designed synthesis of uniformly sized iron oxide nanoparticles for efficient magnetic resonance imaging contrast agents. Chemical Society Reviews. 41 (2012) 2575-2589.

[7] I. Sharifi; H. Shokrollahi; S. Amiri. Ferritebased magnetic nanofluids used in hyperthermia applications. Journal of Magnetism and Magnetic Materials. 324 (2012) 903-915.

[8] P. Md. Gazzali; V. Kanimozhi; P. Priyadharsini; G. Chandrasekaran. Structural and Magnetic properties of Ultrafine Magnesium Ferrite Nanoparticles. Advanced Materials Research. (2014) 128133.

[9] M. Banerjee; N. Verma; R. Prasad. Structural and catalytic properties of $\mathrm{Zn} 1-\mathrm{x}$ $\mathrm{Cu} \times \mathrm{Fe}_{2} \mathrm{O}_{4}$ nanoparticles. Journal of Materials Science. 42 (2007) 1833-1837.

[10] H. El Moussaoui; O. Mounkachi; R. Masrour; M. Hamedoun; E. K. Hlil; Benyoussef, A. Synthesis and super-paramagnetic properties of neodymium ferrites nanorods. Journal of Alloys and Compounds. 581 (2013) 776-781.

[11] S. M. Hoque; C. Srivastava; N. Srivastava; N. Venkateshan; K. Chattopadhyay. Synthesis and characterization of $\mathrm{Fe}$-and Co-based ferrite nanoparticles and study of the $\mathrm{T} 1$ and $\mathrm{T} 2$ relaxivity of chitosan-coated particles. Journal of Materials Science, 48 (2013) 812-818.

[12] I. I. Cabrera; A. Somoza; J. F. Marco; C. J. Serna; M. P. Morales. Synthesis and surface modification of uniform $\mathrm{MFe}_{2} \mathrm{O}_{4}(\mathrm{M}=$ $\mathrm{Fe}, \mathrm{Mn}$, and $\mathrm{Co}$ ) nanoparticles with tunable sizes and functionalities. Journal of Nanoparticle Research. 14 (2012) 1-14.

[13] M. P. Gonzalez-Sandoval; A. M. Beesley; M. Miki-Yoshida; I. Fuentes-Cobas; J. A. Matutes-Aquino. Comparative study of the microstructural and magnetic properties of spinel ferrites obtained by co-precipitation. Journal of Alloys and Compounds. 369 
(2004) 190-194.

[14] K. Petcharoen; A. Sirivat. Synthesis and characterization of magnetite nanoparticles via the chemical co-precipitation method. Materials Science and Engineering: B. 177 (2012) 421-427.

[15] M. C. Mascolo; Y. Pei; T. A. Ring. Room temperature co-precipitation synthesis of magnetite nanoparticles in a large ph window with different bases. Materials. 6 (2013) 5549-5567.

[16] I. Zhang; R. He; H.C. Gu. Oleic acid coating on the monodisperse magnetite nanoparticles. Applied Surface Science. 253 (2006) 2611-2617.

[17] A. Roca; M. Morales; K. O'Grady; C. Serna, Structural and magnetic properties of uniform magnetite nanoparticles prepared by high temperature decomposition of organic precursors. Nanotechnology. 17 (2006) 2783.

[18] J. Vargas; R. Zysler. Tailoring the size in colloidal iron oxide magnetic nanoparticles.

Nanotechnology. 16 (2005) 1474.

[19] W. William. Synthesis of monodisperse iron oxide nanocrystals by thermal decomposition of iron carboxylate salts. Chemical Communications. 20 (2004) 2306-2307.

[20] Q. Lan; C. Liu; F. Yang; S. Liu; J. Xu; D. Sun. Synthesis of bilayer oleic acid-coated Fe 304 nanoparticles and their application in $\mathrm{pH}$-responsive Pickering emulsions. Journal of colloid and interface science. 310 (2007) 260-269.

E-mail:jajeatme@gmail.com, coaquira.ja@gmail.com 